\title{
Research on the Mental Fatigue Evaluation of Modern Manufacturing Operators Based on Bp Neural Network
}

\author{
Ye Lan, Zou Jinhong, Zhang Jie \\ Jiangxi Engineering Laboratory of Digital Manufacturing for Automobile Parts, Nanchang institute of \\ science and technology, Nanchang 330108, P. R. China
}

Keywords: BP neural network, Mental Fatigue of Operators, evaluation

\begin{abstract}
Since modern manufacturing enterprises are supported by advanced science and technology, the production methods and production scope have undergone tremendous changes compared with traditional enterprises. However, in the modern manufacturing enterprises, people are not excluded from the modern production system. After the experimental data are collected and analyzed, BP neural network is used to establish the evaluation model of the fatigue state of the operator. The neural network is trained by the real data and the measures data of the trained neural network verification. The results show that using brain waves and eye movements as evaluation indexes, the evaluation model of mental fatigue state of modern manufacturing enterprises established by BP neural network can accurately determine the operator's mental fatigue state, whose accuracy can reach $93.3 \%$
\end{abstract}

\section{Introduction}

We often say that manufacturing is the transformation of a manufacturing resource into a commodity that can be consumed by people in accordance with the demand of the market. In today's context of industrialization, manufacturing plays an important role in the economic development of our country. It can be said that the development of a manufacturing industry in a country can reflect to some extent the country's productivity level. At the same time, it is widely used in fields such as engineering, astronomy and biology. The advantage of this method is that it has strong non-linear fitting ability. It does not need to give a specific mathematical function in modeling. It can introduce multiple explanatory variables at one time and output multiple estimated values at the same time.

\section{The Modern Manufacturing Enterprise Operator State Simulation Based on Brain Wave}

In the modern manufacturing industry, much manual labor has been replaced by some or all of the machines. Nowadays, what people need to face is to process the feedback information of a large number of manufacturing systems, and must pay special attention to their work and react quickly and accurately based on the feedback information [2]. This feature of the modern manufacturing industry makes the mental load borne by people in the manufacturing system more and more heavy. Therefore, studying the mental workload of the modern manufacturing industry plays an important role in the efficiency of the manufacturing system. And how to accurately judge the fatigue state of the manufacturing system and effectively prevent the fatigue is a difficult problem faced by modern manufacturing industry. In order to correctly evaluate the state of fatigue, we must select the appropriate state characteristics as a judge. Therefore, this paper uses the characteristics of brain waves signal changes to analyze the degree of mental fatigue.

\subsection{The expression of brain fatigue and its influencing factors.}

People are uncomfortable with the subjective feeling of fatigue, do not want to continue to work, hope to get rest and relaxation; The objective performance of fatigue is to reduce the work efficiency, reduce the working ability and decrease the physiological function. Mental fatigue usually occurs during prolonged or overworked work, which leads to mental depression. The main 
symptoms are fatigue, malaise, drowsiness, difficulty concentrating, work efficiency and work quality declining. The most important cause of brain fatigue is the operator's own factors and work environment.

\subsection{The design of Experimental}

\subsubsection{Experimental content}

In order to accurately simulate the contents of the work of modern manufacturing enterprise operators, this paper chooses Psy Tech EP2009 psychological experiment platform to focus attention experiment, cancellation experiment, warning performance test to simulate. The experiment is used to simulate the inspection operations of the modern manufacturing enterprise operator. It can be a systematic safety inspection or a check on the quality of the products, and should be selected for further processing in case of poor performance or defective products. Alert Performance Assignment Assay is a test of the operator's ability to react to a specific phenomenon. The subjective fatigue assessment method used in this article evaluates fatigue, defines a state of non-fatigue with a score of 20 points or less, a state of mild fatigue with a score of 20 points or more but exceeding 60 points, a mild fatigue state with a score of 60 or more The state is severe fatigue.

\subsubsection{Experimental procedure}

1) Fill in the subjective fatigue evaluation form: the operator according to their own subjective feelings, be sure to accurately and accurately fill in the subjective fatigue evaluation form.

2) Simulating the working content of modern manufacturing enterprises Experiment: Open the Ergo Lab human-machine environment synchronization platform, debug brain wave tester and eye tracker, and focus on the sequence of Psy Tech EP2009 psychological experiment platform Experiments (parameters set to rotate: clockwise, speed $5 \mathrm{rpm}$, time: 180 seconds), cancel experiment (parameter set to experimental material: letter B, experiment time: 3 minutes), alerting job performance experiment $20 \%$ for the grid, 2 for the number of experimental units), focus concentration experiment (parameters set to rotate: clockwise, speed $5 \mathrm{rpm}$, time: 180 seconds) Letter B, experiment time: 3 minutes).

3) Highlights Scintillation experiment: The real operator logs on to the Psy Tech EP2009-type psychology experiment platform, and connects the instrument for the bright-spot scintillator experiment. The parameters are set to bright colors: red, the number of experiments: 16 times, the interval time: 1 second.

4) Fill in the subjective fatigue evaluation form: the operator according to their own subjective feelings, be sure to accurately and accurately fill in the subjective fatigue evaluation form.

\section{BP neural network}

\subsection{The basic concepts of neural network}

Studies in modern physiology and anatomy have found that there are about 10 billion neurons in the human brain. One neuron is one nerve cell, 60 trillion synapses and their connectors. In biological terms, neurons are made up of four parts: cell body, dendrites, axons and synapses. Cell body provides the necessary space and energy for the physiological activities of nerve cells and is the most important part of neurons. Cell body in order to get the required material and energy from the outside, will project a lot of nerve fibers outward, these nerve fibers called dendrites, that is dendrites is the cellular material required for the body and energy input. The cell body has a number of outwardly extending nerve fibers, the function of the dendrites opposite, in order to output material and energy, the longest of which nerve fibers called axons, that is, the axon is a cell body material and Energy output. By studying the structure and activity of mimic human brain neurons, researchers established artificial neurons.

The biggest advantage of neural network compared with other algorithms is that the network model can be more in line with the real situation by constantly adjusting the parameters inside the 
network through learning ability. Neural network learning methods are: a mentor to learn, no tutor learning and re-learning. Mentoring learning compares the output with the expected result, and then adjusts the parameters so that the output is closer to the desired result. Without tutor learning is not expected results, but internal learning, self-optimization. Re-encourage learning is not given the desired result, but to give rewards and penalties, learning system self-adjustment.

\subsection{BP Neural Network Model and Structure Algorithm}

The artificial neural network is composed of many basic neurons, and the general model of neuron is shown in figure 1, usually in the form of multiple input and output.

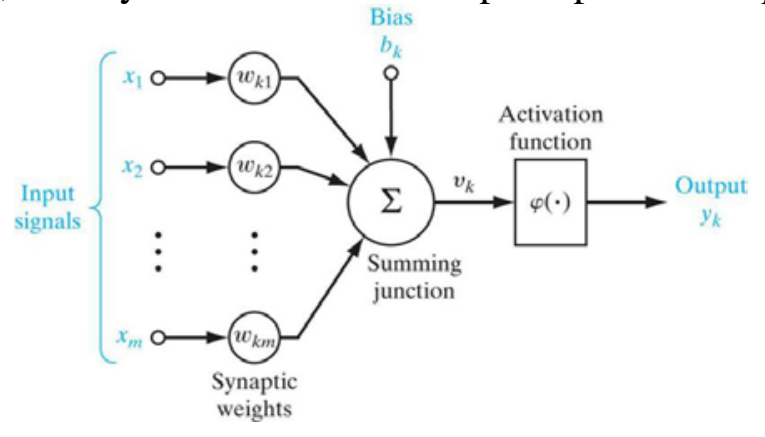

Fig 1 Artificial neuron model

As can be seen from the model, the number of input terminals of BP neurons is not a fixed value, and $\mathrm{m}$ is a natural number greater than or equal to 1 . When the input ends meet the threshold, the input and output relation function is activated, and the information is output at the output end. The training process of BP neural network consists of forward propagation and reverse propagation, and the method to adjust the network parameters is the reverse thrust of error, which is called BP algorithm. BP neural network topology shown in Figure 3, the input vector into the network, the input layer neurons by calculating the hidden layer input, hidden layer using the same method of calculation, the final data is transmitted to the output layer forward, the output vector. After comparing the output with the expected output, if the error exceeds the preset range, the error vector is passed back in the reverse direction.

Using the gradient descent method, the negative gradient of the error function to the neuron's weight or bias, that is, the direction of the error, can be adjusted to the neuron's weight vector and bias vector until the neural network converges. The adjustment of the threshold and weight will be adjusted along the negative gradient of the mean square deviation because the mean square error will drop the fastest and the learning effect will be fastest.
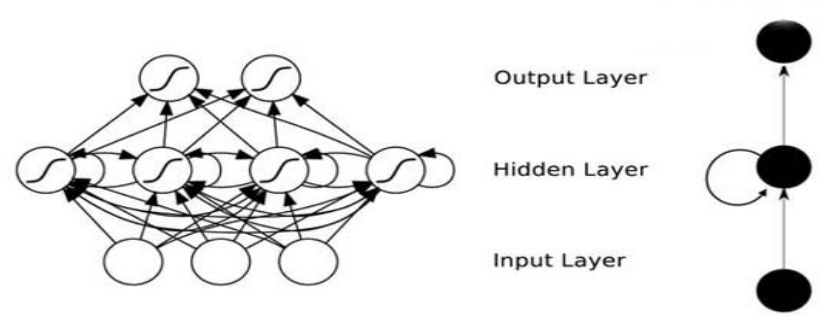

Fig 2 BPNeuron model

$$
x_{k+1}=x_{k}-a_{k} g_{k}
$$

$\mathrm{x}_{\mathrm{k}}$ is the weight of the current weight and the threshold; $\mathrm{g}_{\mathrm{k}}$ is the gradient of the current performance function; $a_{k}$ is the learning rate. The process is as follows.

We take the three-layer BP neural network as an example to illustrate that the change of network layer does not have a substantial impact on the derivation process. Input we use $\mathrm{x}_{\mathrm{j}}$ said that hidden neurons on the layer we use $y_{j}$ said that the output of the output we use $z_{j}$ said. Input information through the role of weight $\mathrm{w}_{\mathrm{ji}}$ transmission hidden layer neurons, hidden layer neurons output through the role of weight $\mathrm{v}_{\mathrm{lj}}$ transmitted to the output. Assuming that the target expected output of 
the BP neural network is $t_{1}$, the following formula is available.

Hidden layer output

$$
y_{i}=f\left(\sum w_{i j} X_{i}-\theta_{j}\right)=f\left(\text { net }_{j}\right)
$$

Among them

$$
n e t_{j}=\sum w_{j i} X_{i}-\theta_{j}
$$

Output output

$$
z_{1}=f\left(\sum j v_{1} y_{i}-\theta_{j}\right)
$$

Among them

$$
\operatorname{net}_{1}=\sum{ }_{j} v_{1 j} y_{j}-\theta_{1}
$$

Output error

$$
E=\frac{1}{2} \sum_{L}(t 1-z 1)^{2}=\frac{1}{2} \sum_{j}\left(t 1-f\left(\sum_{j} v i j-\theta j\right)\right)^{2}
$$

Then the error of the output $\mathrm{E}$ is derived, we can find the weight and the threshold of the gradient, and then the BP neural network weights and thresholds toward the negative gradient direction adjustment, the output error as soon as possible toward the desired The error moves closer until it reaches the set error range.

\section{Establishment of Evaluation Model for Fatigue of Modern Manufacturing Enterprise Operators}

\subsection{Model establishment}

Since it has been decided to use BP neural network as a method of evaluating the fatigue of modern manufacturing enterprises, the next step is to determine the structure of the model. After the model structure is determined, it is necessary to determine the parameters, such as the transfer function used between the layers of the network, set the weight of the initial value of each layer, which learning mode can be used to achieve the best learning efficiency, how much error can be accepted and so on.

\subsubsection{Determine the input neuron.}

How to determine the modern manufacturing enterprise operator fatigue, we must first determine the indicators, there are physiological indicators, such as brain waves, skin potential difference, muscle potential difference, heart rate, etc, there are eye movement indicators, such as pupil diameter size, blink Duration, blink frequency per unit of time, the number of fixation points per unit of time, etc; there are some external physical activities, such as yawning, left and right wait. If all the physiological indicators, eye movement indicators and physical behavior indicators as input neurons, first of all neural network model will be very complicated, computational efficiency will be very low; followed by some indicators of personal habits, personal physiological characteristics Large, sometimes it will reduce the accuracy of the neural network model calculation results. In this paper, the input neurons of brain wave BP neural network model.

\subsubsection{Output neurons to determine}

The output neurons should be the operator's fatigue state. According to the second chapter of this paper, the fatigue state of the operator is divided into three levels, namely fatigue, mild fatigue and severe fatigue, so the neurons of the three states are respectively identified as 100,010,001. 


\subsubsection{Network hidden layer to determine}

Hidden layers are the inner core of neural networks for computations. However, the study of hidden layer shows that the increase of the number of neurons in hidden layer will speed up the operation to some extent, but the increase of the number of layers will also make the calculation of The errors in the reverse transmission process become more complicated. The increase of the number of neuron layers does not improve the precision of the operation. Therefore, the number of hidden layers in the BP neural network model established in this paper is one level.

\subsubsection{Neurons in the hidden layer of the network are identified}

In the BP neural network model, the number of neurons distributed in the hidden layer has a considerable influence on the accuracy of the model operation. When the number of neurons in the hidden layer is relatively large, the learning efficiency of the model will be significantly reduced, and the memory occupied by the model operation will also increase sharply. When the number of neurons in the hidden layer is relatively small, the learning ability of the model will be reduced , Convergence speed will decline.

\subsubsection{Determination of transfer function}

In the BP neural network that is commonly used in transfer function with s-shaped logarithmic function logsig function and logsig function of derived function dlogsig function, s-shaped tangent function tansig function, derived function tansig function dtansig function, pure linear function purelin function, purelin function derived function d purelin function. This paper USES the logsig function.

\subsubsection{Initial weight determination}

Prior to the start of BP neural network training, a set of random numbers is generated by the computer as the initial weights and initial thresholds of the network, and then the thresholds and weights are continuously adjusted after the training starts. Therefore, the initial weight need not be artificial.

\subsubsection{Determination of training samples}

EEG data and eye movement data obtained in this paper are used as training samples. Since the transfer function is determined to be a logsig function, the values of the target vectors are 0.3 and 0.7 , respectively corresponding to 0 and 1 , and can be distinguished from each other by a ratio of 0.5 to 0.5 when outputting. So three training samples can be obtained, one-factor input to the brain wave training samples, eye movement multi-factor input training samples and brain wave multi-factor input eye movement training samples.

\subsection{Model training effect}

After inputting the brain wave data into the untrained BP neural network, the result of the output is Y1 after the MATLAB operation, and the conversion to the fatigue evaluation matrix is the Y11. It can be found that the output result is not only inconsistent with the actual result but also invalid data. After 100 training sessions, as shown in Figure 3, the network reaches the expected error of 0.0001 at the 23rd training. The brainwave data is then input to the trained BP neural network and the output is Y2, which is converted to the fatigue assessment matrix and the expected output T1 is the same.

$$
Y 1=\left[\begin{array}{llllll}
0.4860 & 0.5311 & 0.4427 & 0.8487 & 0.3248 & 0.0124 \\
0.0148 & 0.0125 & 0.0478 & 0.3456 & 0.5485 & 0.9004 \\
0.1882 & 0.2425 & 0.0628 & 0.7545 & 0.6458 & 0.9235
\end{array}\right]
$$




$$
\begin{aligned}
& Y 11=\left[\begin{array}{llllll}
0 & 0 & 0 & 0 & 1 & 0 \\
1 & 1 & 1 & 1 & 1 & 0 \\
0 & 1 & 1 & 1 & 1 & 1
\end{array}\right] \\
& Y 1=\left[\begin{array}{llllll}
0.6860 & 0.3211 & 0.3282 & 0.3426 & 0.3018 & 0.2924 \\
0.3148 & 0.6182 & 0.6778 & 0.6416 & 0.3085 & 0.3004 \\
0.3002 & 0.3005 & 0.3012 & 0.3125 & 0.6958 & 0.7235
\end{array}\right] \\
& T 1=\left[\begin{array}{llllll}
0.6 & 0.3 & 0.3 & 0.3 & 0.3 & 0.3 \\
0.3 & 0.8 & 0.8 & 0.8 & 0.3 & 0.3 \\
0.3 & 03 & 0.8 & 0.8 & 0.3 & 0.8
\end{array}\right] \\
& \text { EMSE }
\end{aligned}
$$

Fig 3 BP Neural Network Error Performance Curve

\section{Conclusions}

BP neural network is used to establish operator fatigue state and BP neural network evaluation model of modern manufacturing enterprise. Through training analysis and BP neural network evaluation model of brain wave, the computational efficiency is more efficient. Then, the model of BP neural network for fatigue testing of modern manufacturing enterprises is validated. The accuracy and efficiency of the results are far higher than those of subjective fatigue assessment, and the accuracy of the results is $93.3 \%$.

\section{Acknowledgments}

This work was supported by the Nanchang Institute of Science and Technology research project for Jiangxi Engineering Laboratory of Digital Manufacturing for Automobile Parts under Grant No. SZZX-17-18.

\section{References}

[1] Ayaz H, Willems B, Bunce B, et al. Cognitive workload assessment of air traffic controllers using optical brain imaging sensors[C]. Advances in Understanding Human Performance: Neuroergonomics, Human Factors Design, and Special Populations. New York, USA,2010: 21-31.

[2] Rice S, Trafimow D, Time pressure heuristics can improve performance due to increased consistency[J]. The Journal of General Psychology,2012,139(4): 273-288

[3] Grillon C, Quispeescudero D, Mathur A, et al. Mental fatigue impairs emotion regulation[J]. Emotion,2015,15(3):383-389.

[4] Mun S, Kim E S, Park MC. Effect of mental fatigue caused by mobile 3D viewing on selective attention: an ERP study[J]. International Journal of Psychophysiology,2014, 94(3): 373-381 